\title{
The Appropriate Technology of Vertical Greening of Residential Buildings in South China
}

\author{
LIN Jing ${ }^{1, a}$ \\ ${ }^{1}$ China Academy of Building Research, Shenzhen 518000 \\ a164957473@qq.com
}

\begin{abstract}
Keywords: Residential Building, Vertical Greening, Appropriate Technology
Abstract: Residential buildings closely relating to people's life have special design requirements in vertical greening of building elevation. The paper introduced types of vertical greening technology on the basis of studying on the residential buildings in southern China area, and then discussed on suitable technical of vertical greening when designed balconies, opening frameworks and outer wall of building elevation, which was to provide reference for the feasibility of the vertical greening of residential buildings.
\end{abstract}

\section{Introduction}

Vertical greening technology is more used in public buildings, commercial buildings and tourist attractions and others than in residence in southern China and even in the whole of China. However, some serious environmental problem such as the decreasing city planting area and worsening urban heat island greatly affect citizen and ecological balance. Suitable technical of vertical greening utilized in residence will help to improve the air quality of residential area, reduce the heat island effect and increase the comfort of indoor environment to achieve sustainable development of modern cities.

\section{Vertical greening system}

Vertical Greening is a form that all kinds of plants are formed vertical surface by means of clinging, fixing, affixing and hanging on building facades or other structures [1]. Depending on the difference between vertical green growth substrate and planting methods, Vertical greening system is divided into four types, including climbing system, carrier system, modular system and hydroponic system.

\section{Climbing system}

With adsorption and hanging characteristics of climbers, vines are planted on the base of linear or net structure of the building with vertical greening of climbing system. It is divided into natural climbing system and auxiliary climbing system according to whether it contains auxiliary structures. Auxiliary climbing system is classified into systems of cable, wire mesh and grid [2]. The cable system is fixed rope or steel on the wall of the building in horizontal or vertical direction for climbing plant and controlling growth orientation and morphology, which applies to vines with fast growth and dense leave. The wire mesh system forming a plane grid on the walls of buildings is more flexible than the cable system. The specification can be customized in advance according to the size of various vines, which is suitable for plants of slow growth. The grid system is to constitute a climbing frame with which vines climb along the plants by the weld metal mesh, mosaic panel or wooden frame and other auxiliary components (Figure 1). The cable system and the wire mesh system constitute auxiliary bracket in the plane while the grid system constitutes in the three-dimensional structure in order to prevent destructing the building wall availably.

\footnotetext{
${ }^{1}$ LIN Jing, (1986- ) , Female, Master, Intermediate engineer, E-mail: 164957473@qq.com, Address: The 6 floors, Fucheng technology Building, Gaoxin Road, Nanshan District, Shenzhen City, P.R. China 


\section{Carrier system}

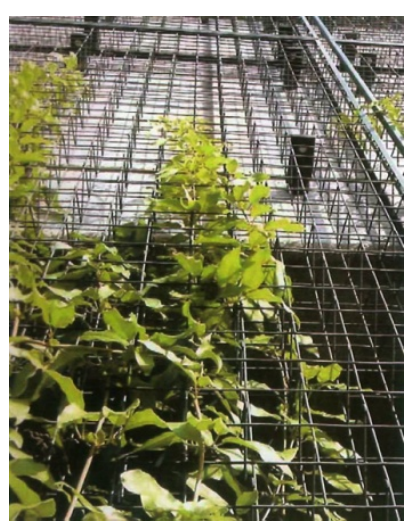

Figure 1 Climbing system

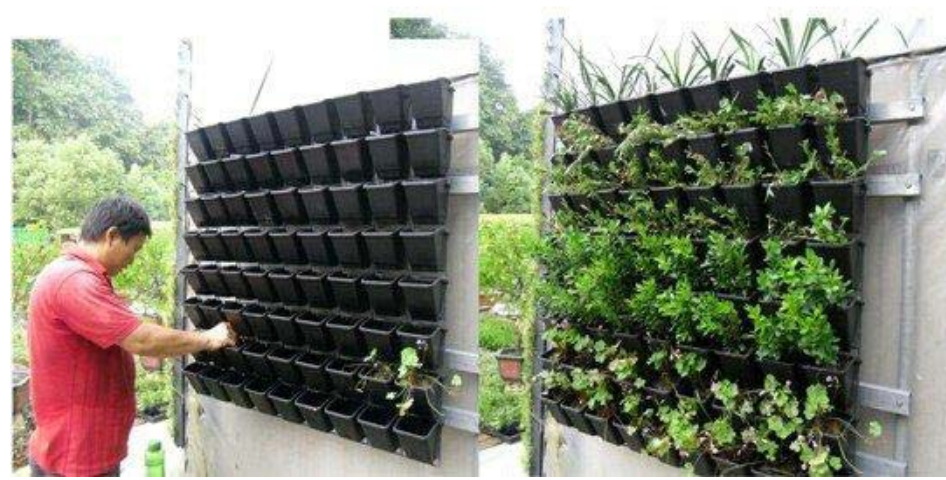

Figure 2 Carrier system

Carrier system is the greening formation that each plant individually planted in different containers which substantially parallel to the ground, placed or hung on the supporting frame made of stainless steel, reinforced concrete or other materials (Figure 2). Drip irrigation and spray irrigation system are generally used with a water pipe with high efficiency allowing the water to flow from top to bottom through the entire wall.

\section{Modular system}

Modular system mainly consists of three parts: the greening module, the structure system and the irrigation system. The Figure 3 shown greening module form a green surface by fixed to the buildings with system structure and support frame connecting planting panel and building walls. In general, corrosion resistance and highly intensive stainless steel frame are selected to well meet the requirements to ensure safety of plant panel and firm connection. The arrangement of irrigation system is consistent with frame structure which is convenient to provide water or liquid fertilizer to each unit module [3].

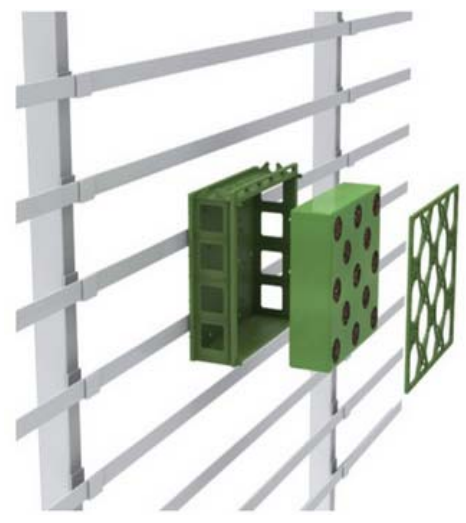

Figure 3 Modular system

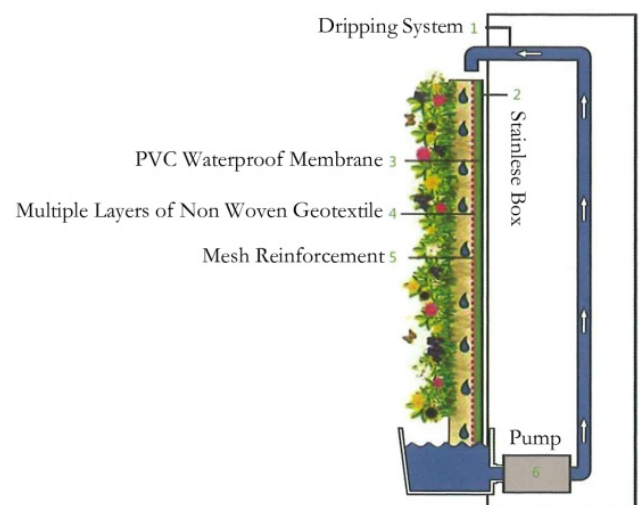

Figure 4 Hydroponic system

\section{Hydroponic system}

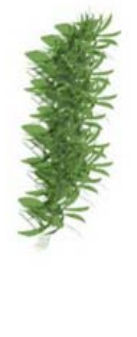

France Patrick Blanc firstly used approach of hydroponic system. Metal frame is erected on the structure wall separated from the wall surface and form an air layer to well play a role in keeping warm and sound insulation. The polyvinyl chloride board is nailed on the metal frame to form a layer of waterproof layer which fixed two layers of felt with steel nail. The surface layers of felt are cut and roots of the plants placed at these openings of felt, and then the plants grow in the two layers felt. Automatic irrigation equipment above the frame fertilize regularly from top to bottom, nutrition solution expand along felt to provide plant essential nutrients (Figure 4).

\section{Vertical greening of residential building in south China}

With hot and humid climate in southern China, suitable vertical greening fixed up on balconies, opening frameworks and outer wall of residential building can not only improve the living environment and microclimate of resident but also beautifies and activates the building facade. The selection of vertical greening technology should follow the principles of applicability, economy, ecology, aesthetics and sustainability. 


\section{Balcony greening}

In the urbanite of the life in ferroconcrete city, the balcony is the important media residents connecting with outside natural world. Adding vertical greening into balcony can decorate the building's interior environment as well as giving pleasure. Layout options of residential buildings in southern China mainly distribute in the north-south direction with the characteristics of southern balconies with long sunshine and good ventilation. Climbing system and Carrier system are easy to maintain and manage so as to choose for balcony vertical greening. Plants are advised to select Shallow-rooted plants with features of heliophyte, drought and heat resistance, well-developed horizontal root system and extensive management and some small herbaceous vines or flowers. The following shows three types of vertical greening with low maintenance and management and better landscape effect.

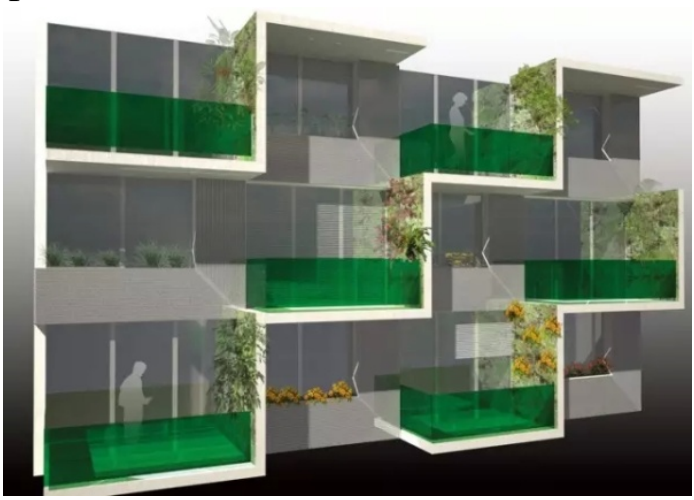

Figure 5 Balcony with carrier system

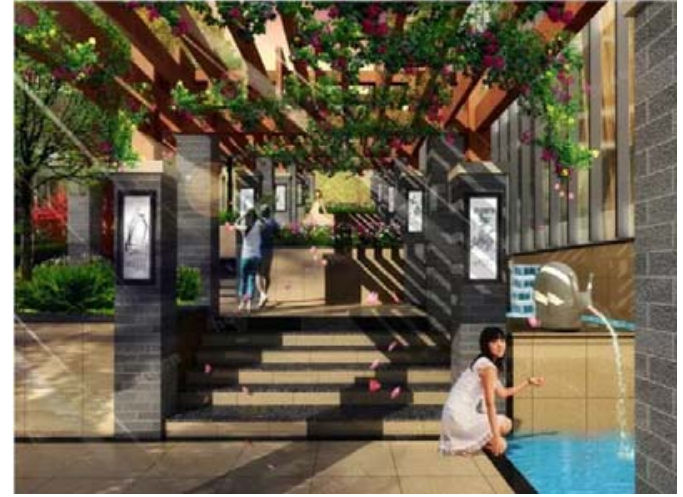

Figure 6 Carrier system on opening framework

The first method is that plants wind and cling to the balcony fence, invisible anti-theft network or erection of scaffolding form. Vines planted in containers or planters climb following veranda fence and invisible network security and form green screen. Climbing and twining vines for the balconies commonly used such as Ficus pumila and Hedera nepalensis var. sinensis, some vines with flowers including Ipomoea nil, Wisteria sinensis, Ipomoea quamoclit, Rosa chinensis and so on, some vines with ornamental fruits are chosen such as Vitis vinifera and Luffa aegyptiaca.

Another method is that plants planted in the planting groove of the balcony grow downward to exhibit green curtain. There some vines are well applied such as Wisteria sinensis, Jasminum mesnyi, Ipomoea nil, Hedera nepalensis var. sinensis, Rosa chinensis, Bougainvillea glabra and so on.

The third method is to build a vertical support frame in corner or against a wall of the balcony, and each individual plant is placed on it by maintaining level, which saves the active space of the balcony and achieves good energy saving effect with effective architectural design. The L-shape balcony with ladders as Figure 5 showed can produce a chimney effect that hot gas escapes indoor and lower the temperature, meanwhile, it can reduce the energy consumption of the refrigeration equipment in summer.

\section{Vertical greening of the opening framework}

Opening framework which comes from arcade of Lingnan architecture and generally appears in residential buildings in southern China is an open public space of the building supported by pillars. Setting on the bottom or between shops and residential layers, vertical greening of the opening framework can separate different functional areas, benefit to residential air circulation avoiding indoor humidity and provide a neighborhood contact space for the residents. In addition, as supplement and extension of residential green, it can not only soften and divide the space but beautify and improve the environment of the overhead layer, and then increase the level sense of the space and interest. Channel wind is easy to form because of lacking of sunlight in opening framework, therefore, plants are advised to choose with shade resistant and good wind resistance. The selection of plants is different according to the different direction of opening framework. Space towards south has the most direct light and the longest sunshine duration, shade-tolerant plant should be chosen such as Parthenocissus quinquefolia, Juniperus chinensis 'Kaizuka', Mimosa pudica, Agave sisalana and Ficus pumila; the space towards east or west mostly has diffusion light 
except less direct light, relative shade tolerance plants will be chosen such as Pilea notata, Excoecaria cochinchinensis, Rhododendron simsii and Mahonia fortunei; the space towards north or position in the corner has little light, skiophyte such as Adiantum capillus-veneris, Nephrolepis cordifolia, Maranta arundinacea, Alocasia odora and Monstera deliciosa; plants with shade resistant and good wind resistance are planted towards west or north, such as Dracaena deremensis 'Virens Compacta', Syngonium podophyllum, and Caryota maxima [4].Type of vertical greening is different according to the space environment and functional requirements of opening framework, the following summary two vertical green forms with low cost, good effect and long life.

One is to plant vines around the pillar of the opening framework. Planting in land as far as possible is benefits to plant growth and maintenance and management which reduce costs. Natural climbing and auxiliary climbing are adopt by wirerope or cable and wire mesh systems with wirerope. Such plant like Euonymus fortunei, Parthenocissus tricuspidata, Hedera nepalensis var. sinensis and Epipremnum aureum can be well chosen.

Another from is setting up canopy frame at the top of opening framework, which extends outdoor landscape into opening framework and creates a specific landscape atmosphere. As shown in Figure 6, shade tolerant vines are selected such as Euonymus fortunei and Epipremnum aureum.

\section{Vertical greening of exterior wall}

Vertical greening is one of the most direct ways to green the outside wall of residential building and it is also one of the most effective ways to improve the quality of living environment. Climate in southern China is mainly damp and hot, indoor temperature is usually high and heat radiation is not good influenced by west sunshine and wind direction in south and southeast. Therefore, sunshade and heat preservation facilities setting on east-west exterior window and wall respectively should be designed to save energy and improve the indoor environment. Wall vertical greening has a significant effect on reducing temperature of exterior wall and indoor environment, solar radiation intensity and improving the surrounding relative air humidity. According to the measured results showed by company Hannor: cooling effect is obvious as using vertical greening of exterior wall. With $38^{\circ} \mathrm{C}$ of the outdoor temperature, temperature of the exterior wall painted deep gray coating without vertical greening reaches up to $50^{\circ} \mathrm{C}$, whereas there is $27^{\circ} \mathrm{C}$ with vertical greening; relative air humidity increases $10 \%$ to $20 \%$ around exterior wall with vertical greening; indoor temperature decreases about $3-5^{\circ} \mathrm{C}$ obviously. According to direction and position of the wall, the form of can be set to following types to achieve different functions and effects.

\section{Vertical greening on exterior wall of east-west direction}

Setting vertical greening on exterior wall of east-west direction of residential building can reduce temperature of the surface of wall and indoor environment. Residential construction in southern China is mainly toward the north-south direction and seldom set bedroom, living room and other main functional room with small size of the window in east-west direction. Climbing system of vertical greening can be made by means of piecewise planting with linear or reticular structure. Natural climbing system without auxiliary frame is not to advice to adopt with the reason of less control of vines direction and damage for buildings. Plants with heliophyte, drought and heat resistance should be chosen such as Parthenocissus tricuspidata, Ficus pumila and Lonicera maackii, while Parthenocissus tricuspidata with fast growth and more branches is suitable to plant on west wall.

In Fengjing residence of China Vanke, vines are dragged by steel cable on exterior wall, planting groove are set every two layers to plant vines like Lonicera maackii and Quisqualis indica, which reaches climbing height of $8 \mathrm{~m}$ and form a good landscape effect (Figure 7). Auxiliary frame with wire mesh system should be to adjust the size and density of grid according to plant type and effect of landscape design. 

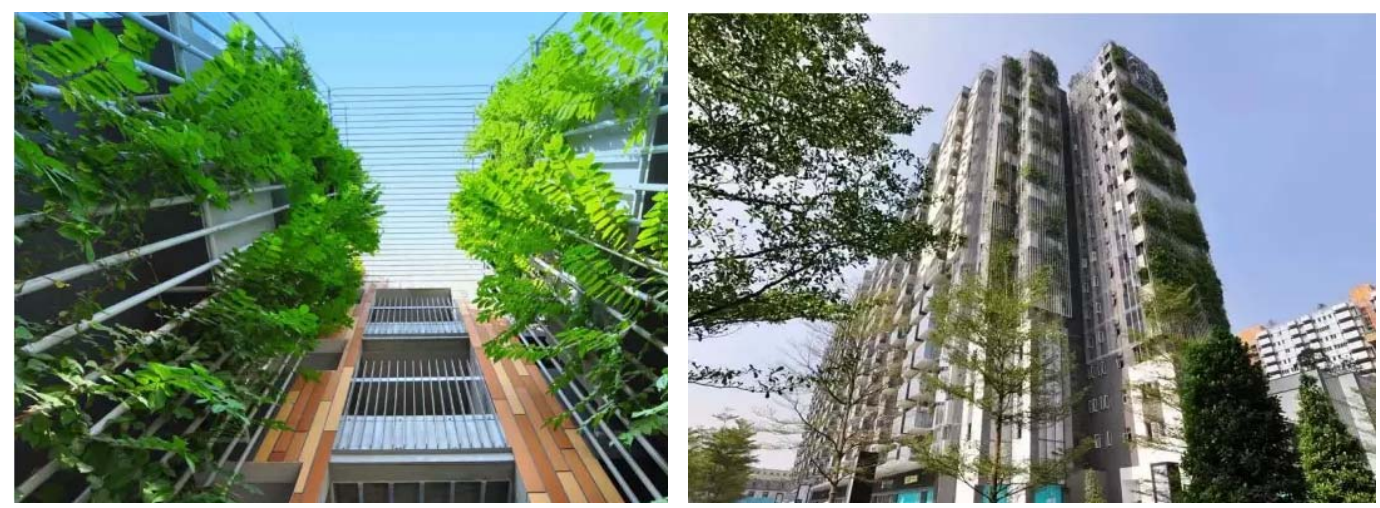

Figure 7 Cable system on east-west exterior wall of buildings

\section{Vertical greening on the wall of main entrance of buildings}

Vertical greening set on the wall of main entrance of buildings can give a person a deep first impression and intuitive visual perception, therefore it is high requirements in aesthetic character of the vertical greening. The selection of plants should be different according to effect of landscape design, the direction, the position and vertical greening systems.

One method is to set up steel wire for exterior wall of entrance of ground floor and plant vines in land. Transparent glass of lobby exterior wall in entrance of first floor is usually used in order not to affect natural illumination and sight line of indoor to outdoor. Moreover, separation distance of steel wire cable assisting Climb and guide should not be set too close.

Another method is to add structural framework on exterior wall of residential entrance embed the plant module in the frame. Using for high facade of residential building, it is easy to form different patterns. The structure and plant assembled with unit element are convenient to exchange problematic module unit, but the cost is higher than climbing system.

When design limits bearing of building facade and requires higher aesthetic character, soilless culture can be used for vertical greening (Figure 8). In soilless culture, supporting frame with blankets and non-woven fabric as containers is set on wall surface, nutrient solution is used as growth medium and automatic irrigation system is equipped. Pteridophyta, epiphyte and hemiepiphyte cultivated without soil, lithophytes and plants without roots forcing through the interlining should be chosen such as Nephrolepis cordifolia, Asparagus cochinchinensis, Spathiphyllum kochii, Sansevieria trifasciata, Schefflera octophylla, Dianella ensifolia, Tradescantia spathacea, Tradescantia zebrine, Commelina purpurea, Trachelospermum jasminoides and Epipremnum aureum. This method breaks through the limitation of pattern of module with greater freedom of plant type and modeling, which has a better associative perception. However, the prime cost is higher as well as maintenance and management.
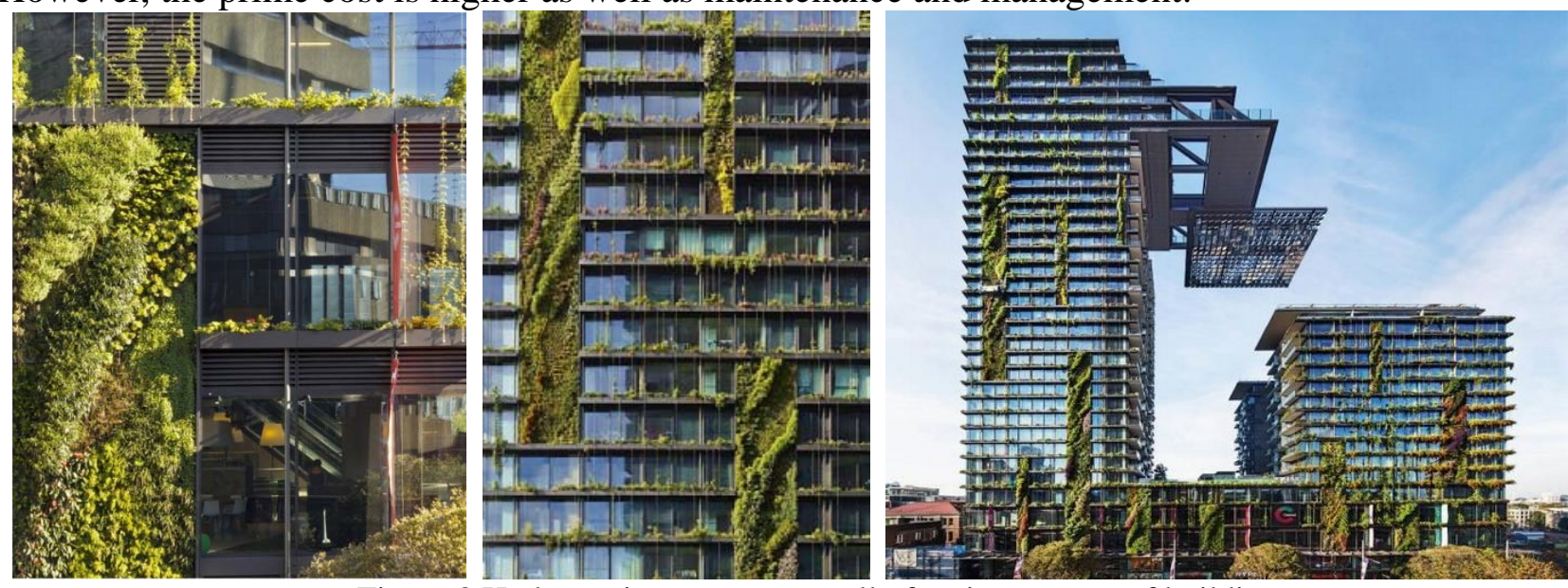

Figure 8 Hydroponic systems on wall of main entrance of buildings

\section{Conclusions}

With advantage of simple technology, low cost, high survival rate and easy maintenance and management, vertical greening of climbing system is suitable to popularized and applied in the residential building. But it also has weakness with limited climbing height and coverage area of 
vines, monotonous greening effect, long time to form the green wall and difficult replacement. Therefore, segmented planting in every certain height and mixed system with more than two kinds of vertical greening are advised such as combinations of climbing system and carrier system, combinations of climbing system, modular system and hydroponic system, which adopts its strong points while overcoming its weak points and rich facade landscape. Carrier system with convenience of maintenance and management and replacement of potting is suitable for residential balcony and windowsill. Modular system with easy installation can be arbitrarily assembled, but need to add framework on exterior wall and from thick green wall. Hydroponic system with frivolous module has high technology and complicated service if occurring with leakage, damage of water barrier and other issues. The last two systems have higher cost in earlier stage and maintenance and management than the two former, but it can be carried out on a large area of vertical greening with superior difficulty and form the ensemble landscape. The actual project can be based on the reasonable choice of project conditions.

\section{Acknowledgements}

This work was financially supported by Young Scientific Research Fund of China Academy of Building Research.

\section{Reference}

[1] Keyan Niu, Xiaosong Shi. Roof garden and vertical greening. Beijing: Chemical industry press, (2011)

[2] Jialing Tong (editor), Wenwen Yin (translater). Living wall. Shenyang: Liaoning science and technology publishing house (2013)

[3] Junhui Fu. Preliminary studies on living wall model in Beijing. Beijing Forestry University (2011)

[4] Yang yang. Research on design of open floor in high-rise residential building of south China. South China University of Technology (2011) 